\title{
The Spatial Distribution of Colonial Food Provisioners
}

\author{
Reuven Dukas* and Leah Edelstein-Keshet \\ Institute of Applied Mathematics, University of British Columbia, Vancouver, BC V6T 1Z2, \\ Canada
}

(Received on 9 April 1997, Accepted in revised form on 11 August 1997)

\begin{abstract}
We present spatially explicit models to predict the optimal distribution of numerous food provisioners that share a nesting aggregation. We compare predictions for solitary and social provisioners, and for three foraging currencies. When distinct food patches with identical food parameters are considered, fewer provisioners use the farther patches. The rate of decline in number of provisioners depends on the currency used. With net rate of energy intake (net energy gain over time), the rate of decline is relatively small under a wide range of realistic parameter values and distances; with efficiency (net energy gain over energy cost) and empirically derived energetic costs, the rate of decline is much higher. With lifetime fitness (lifetime food delivery under predation risk), outcomes depend on the ratio of mortality risk during flight and during food collection at the patch. With all currencies, there is a difference between the optimal spatial distribution of solitary and social provisioners, with a larger proportion of social provisioners being farther from the central place. The optimal distribution of solitary provisioners in a two dimensional field of uniformly distributed food such as a flowering meadow is such that the density of solitary provisioners (number per unit area) declines monotonically with distance, and provisioner number peaks at half the maximum area used.
\end{abstract}

(C) 1998 Academic Press Limited

\section{Introduction}

Many animals nest in aggregations consisting of numerous individuals. For example, various solitary bees form aggregations of thousands of individuals, with the largest recorded aggregation containing about 12 million nests (Batra, 1984); a single honey bee colony may have more than 60000 bees (e.g. Seeley, 1995); and some sea bird colonies, such as those of thick-billed murres have up to 250000 nests (e.g. Gaston et al., 1983). Causes for such aggregation are limitation of appropriate nest substrates such as suitable islands for many sea birds, caves for bats, and certain soils for solitary bees, defense against predators, or communication about food sites (e.g. Michener, 1974, Brown \& Brown, 1996).

\footnotetext{
*Author to whom correspondence should be addressed. Present address: Nebraska Behavioral Biology Group, School of Biological Sciences, University of Nebraska, Lincoln, NE 68588-0118, U.S.A.
}

The concentration of numerous food provisioners in a single location must have dramatic effects on the spatial distribution of the provisioners while they are collecting food, and on the food itself. This general notion has been recognized for decades by economists and ecologists [reviewed by Hamilton \& Watt (1970), and Covich (1976)]. However, explicit evaluations of the spatial distribution of non-territorial food provisioners that nest in large aggregations are few and incomplete: Hamilton et al. (1967) discussed factors determining daily dispersal of non-provisioning starlings from a single winter roost. They argued that the fitness value of feeding at varying distances from the roost should be identical because of reduced starling density and thus increased food intake with increased distance. Covich (1976) focused on the shape and size of a single territory and a set of neighboring territories. Taylor (1978) presented the first formal model for the optimal allocation of colonial foragers between two food sources and tested qualitative predictions with ants (Taylor 1977). 
Although the parameter values needed for quantitative predictions did not exist in the 1970s, some of these parameters are readily available today in studies on the determination of individual behavior in animals including colonial nesting birds and bees (e.g. Welham \& Ydenberg, 1993; Ydenberg \& Schmid Hempel, 1994).

Anderson (1978), Orians \& Pearson (1979), and Schoener (1979) considered optimal behavior of a single central place forager. Their models capture the notion of the greater cost of traveling farther from the nest, but they do not address issues of interactions among a large number of foragers from a single central place. In contrast to the optimality approach taken in the above papers, Seeley and colleagues (Camazine \& Sneyd, 1991; Seeley et al., 1991; Bartholdi et al., 1993) have focused on models of the mechanisms underlying allocation of honey bee foragers among food patches. Although this mechanistic approach is revealing, a general ultimate model providing optimality predictions can contribute further insights about how social or solitary food provisioners sharing an aggregation should distribute themselves in space.

Recently, variations of the ideal free distribution (IFD) model have become prevalent in studies on the spatial distribution of foragers including social bees. In its basic form, the IFD assumes that (i) renewable food is available at different rates in distinct patches, (ii) foragers are ideal in that they know exactly the rates of food deliveries, (iii) foragers are free to choose any patch with no interference competition, and (iv) other factors such as predation do not influence patch choice. All of these assumptions and additional factors, including costs of travel between patches, perceptual biases, and learning, have been addressed in analyses subsequent to Fretwell \& Lucas' (1970) original formulation of the IFD [reviewed by Milinski \& Parker (1991), Kacelnik et al. (1992) and Sutherland (1996)].

In order to apply IFD ideas to the foraging of colonial breeders, one must assume that the locations of and distances between food patches and foragers' nests do not affect foragers' spatial distribution, competitive abilities, or food preferences. Indeed a few studies on bees have made this assumption quite explicitly (Pleasants, 1981; Possingham, 1992). Although this is a legitimate theoretical assumption, it is obvious that a nest's spatial location can affect aspects of provisioner's foraging. Hence spatially explicit models are needed to analyse the distribution of provisioners that nest in large aggregations.

Here we analyse the spatial distribution of numerous individual provisioners that use the same central place. Specifically, we address the following questions: first, how should either solitary or social provisioners from a single aggregation distribute themselves among distinct food patches at varying distances from the aggregation? Second, how does the foraging currency used (rate or efficiency maximization) affect predictions on the spatial distribution? Third, how do predictions change when predation risk is considered? Fourth, what is the optimal spatial distribution of provisioners in a two dimensional field of food? Throughout our presentation, we use bees for our numerical illustrations because bees are ideal for empirical evaluation of our assumptions and predictions. Even so, our models are general and thus relevant for any aggregation of solitary or social food provisioners such as ants and birds (Holldobler \& Wilson, 1990; Brown \& Brown, 1996).

\section{The Model}

\section{PROVISIONER DISTRIBUTION AMONG DISTINCT PATCHES}

In this section, we derive the general equations for evaluating the optimal subdivision of provisioners among distinct patches that differ in their distances from the provisioners' aggregation. First, we derive the equations for rate and efficiency maximization; then we determine the algorithms for solitary and social provisioners. The derivation below considers all the realistic parameters needed for modeling the distribution of colonial food provisioners. The cost of this biological realism is that it does not allow easy analytic solution. Hence, in Appendix A, we develop a somewhat simpler model and include analytic results.

\section{Rate of energy intake}

When the provisioner collects food for delivery and acquires the same food to meet its own energetic needs, we can consider the average long term net rate of energy delivery per trip, which is the total energy gained over the total time spent flying to and from food patch $i$ :

$$
\mathrm{R}_{i}=\frac{L e_{i}-C_{f i}}{t_{p i}+t_{f i}}
$$

where $L$ is the maximum load capacity in units of volume (or weight), $e_{i}$ is the energy content per unit volume (or weight), $C_{f}$ is the total energetic cost of flight, $t_{p i}$ is the total time spent in patch $i$, and $t_{f i}$ is the total time spent flying to patch $i$ (see Schmid-Hempel et al., 1985). For simplicity, we assume here that the time and energy cost of unloading food is negligible. 
The overall energetic cost of flight is $C_{f_{i}}=t_{f i} c_{f}$, where $c_{f}$ is the cost of flight per time unit. The total time spent flying to patch $i$ and back is $t_{f i}=2 d_{i} / v$, where $d_{i}$ is the distance to patch $i$ and $v$ is flight velocity. If the provisioner loads a full load, then its patch duration is $L e_{i} / r_{i}$, where $r_{i}$ is the net rate of loading in patch $i$. Substituting the above parameters in (1), we get

$$
\mathrm{R}_{i}=\frac{L e_{i}-\frac{2 d_{i}}{v} c_{f}}{\frac{L e_{i}}{r_{i}}+\frac{2 d_{i}}{v}} .
$$

The net rate of loading in patch $i$ can be described as

$$
r_{i}=\frac{p_{i} e_{i} s_{i}}{s_{i}+n_{i}^{q}}-c_{p}
$$

where $p_{i}$ is the gross maximum per capita rate of loading in patch $i$ [volume (or weight) per unit time], $s_{i}$ is a parameter related to patch size defined in units of number of provisioners, $n_{i}$ is the number of provisioners foraging simultaneously in patch $i, q$ is the interference competition $(q \geq 1)$, and $c_{p}$ is the energetic cost of loading while in the patch (energy per time unit).

Equation (3) relates food production rate in a patch to the actual net loading rate experienced by a provisioner as affected by the total number of provisioners using that patch and the way they interact. The gross loading rate is determined by four variables. With almost no provisioners in the patch $\left(n_{i} \rightarrow 0\right)$, the rate is approximately $p_{i} e_{i}$. For $n_{i}>0$ and $q=1$ (no interference), loading rate is affected by $n_{i}$ and by $s_{i}$, which can be perceived as an indirect measure of patch size; for example, $s_{i}$ can be related to the number of open flowers in a patch. In the case of no interference, $s_{i}$ is the number of provisioners at which per capita gross consumption rate is half its maximum rate (i.e. $p_{i} e_{i} / 2$ ). With $q=1$, eqn (3) depicts exploitation competition only; with $q>1$, we have both exploitation and interference competition. Note that we assume here no patch depletion. We chose eqn (3) to depict net consumption rate because it is a simple yet biologically meaningful function. While it is not directly based on empirical data, it is in agreement with other functions used to depict exploitation and interference in theoretical and empirical studies (Hassell, 1978; Possingham, 1992; Sutherland, 1996). Note that for completeness, we included $q$ as the parameter for interference competition; however, for brevity, we assumed no interference (i.e. $q=1$ ) in our numerical examples below.

\section{Efficiency}

Empirical studies in birds and bees suggest that efficiency, defined as the ratio of net energy gain over energy cost, better describes individual behavior than net rate of energy gain [eqns (1) and (2); Schmid-Hempel et al., 1985; Ydenberg, 1997]. We thus compared solutions using rate as the currency with those using efficiency. For efficiency, we modified the denominator in eqn (2) to convert patch and flight duration into energetic costs:

$$
\mathrm{E} f f_{i}=\frac{L e_{i}-\frac{2 d_{i}}{v} c_{f}}{\frac{L e_{i}}{r_{i}} c_{p}+\frac{2 d_{i}}{v} c_{f}} .
$$

Above we described the spatially explicit foraging currencies required for calculating provisioner distribution. We now turn to evaluate the distinct algorithms needed for predicting the spatial distribution of solitary and social provisioners. Here "social" refers to a colony such as in social hymenoptera, where a colony's fitness is determined by the cooperative effort of all its members.

\section{Solitary provisioners}

If the foraging arena comprises several patches at varying distances, then at equilibrium, the rate of energy intake by (or efficiency of) a forager at any of the patches for which $n_{i} \geq 1$ should be identical (Hamilton et al., 1967):

$$
\mathrm{R}_{1}=R_{2}=\ldots=R_{i}
$$

where $R_{i}$ is as defined in eqn (2), and

$$
\sum_{i} n_{a i}=\sum_{i} n_{i}\left(1+\frac{t_{f i}}{t_{p i}}\right)=N
$$

where $n_{a i}$ is the number of provisioners using patch $i$, and $N$ is the total number of active foragers in the aggregation. Equation (6) means that the total number of foragers using all available patches (including the foragers on the way to and from the patch) equals the number of foragers available. This equation also illustrates the important point that the number of foragers observed simultaneously in a patch $\left(n_{i}\right)$ can be much smaller than the number of foragers using the patch $\left(n_{a i}\right)$, especially for patches far from the nest, where flight duration $\left(t_{f}\right)$ may be long in relation to duration in the patch $\left(t_{p i}\right)$. For example, for a patch for which flight duration is identical to trip duration, only half the number of provisioners using that patch will be observed at the patch at any given time. 


\section{Social provisioners}

In this case, the allocation of foragers among patches should be such that the overall rate of energy intake (or efficiency) of the colony, $R_{c o l}$, is maximized. That is, the overall rate is the total food collected by all members of the colony over the sum of their foraging duration:

$$
\mathbf{R}_{c o l}=\frac{\sum_{i} n_{a i}\left(L e-\frac{2 d_{i}}{v} c_{f}\right)}{\sum_{i} n_{a i}\left(\frac{L e_{i}}{r_{i}}+\frac{2 d_{i}}{v}\right)}
$$

subject to the constraint expressed in eqn 6 . Equations analogous to (5) and (7) can be derived for the efficiency currency.

\section{BALANCING FORAGING GAIN AND PREDATION RISK}

While the above rate and efficiency currencies consider only rates of energy gain and cost, it is clear that trade-offs between food gain and mortality rate underlie many foraging decisions (e.g. Sih, 1980, 1992; Werner \& Gilliam, 1984; Lima \& Dill, 1990). We thus extend our analysis to include predation risk, and derive equations for lifetime fitness for solitary and social provisioners. We define $\mu_{f}$ and $\mu_{p}$ as the probabilities of mortality per time period during flight and in the patch respectively. Hence, the probability of survival per provisioning trip is

$$
\sigma_{T i}=\left(1-\mu_{f} t_{f i}\right)\left(1-\mu_{p} t_{p i}\right)
$$

(with $\mu_{f} t_{f} \ll 1$ and $\mu_{p} t_{p i} \ll 1$ ). We assume that lifetime energy gain is closely related to lifetime reproduction in solitary provisioners and to the contribution of a social provisioner to its colony's fitness. Clark \& Dukas (1994) compared fitness of a social provisioner such as a social bee for which fitness is incremented each provisioning trip to the fitness of a solitary provisioner such as a solitary bee, which increments fitness only when she successfully completes provisioning a cell. We use here a similar approach in order to compare optimal spatial distribution of solitary and social provisioners. In either case, we assume that the provisioner always uses the same patch, with net energy gain per trip, $\phi_{i}$, as before:

$$
\phi_{i}=L e_{i}-\frac{2 d_{i}}{v} c_{f} .
$$

We also assume constant survival probability per trip, constant food gain, and an unlimited number of potential foraging trips.

\section{Social provisioners}

With the above assumptions, the lifetime contribution to colony fitness of a social provisioner (Clark \& Dukas, 1994) is

$$
\mathrm{F}_{s o c i}=\sigma_{T i} \phi_{i}+\sigma_{T i}^{2} \phi_{i}+\ldots=\frac{\sigma_{T i} \phi_{i}}{1-\sigma_{T i}} .
$$

If survival probability per trip is high, eqn (9a) can be approximated by

$$
\mathrm{F}_{s o c i}=\frac{\phi_{i}}{1-\sigma_{T i}} .
$$

Note that maximization of eqn (9b) is related to minimization of $\mu / g$ (mortality rate over growth rate) as used in analyses of ontogenetic habitat shift (Werner \& Gilliam, 1984; Gilliam \& Fraser, 1987; Clark \& Dukas, 1994). Also, note the similarity between eqn (9b) and the efficiency algorithm [eqn (4)]. The two equations share the numerator, which is net energy gain per trip; the denominators of each equation involve cost, energy expenditure per trip for efficiency, and probability of mortality per trip for lifetime gain.

For the sake of comparison with solitary provisioners, we wish to quantify the contribution of a cohort of social provisioners to their colony's fitness, $F_{c o l}$. If the foraging arena comprises several patches at varying distances, the distribution of social provisioners among these patches should result in the maximization of

$$
\mathrm{F}_{c o l}=n_{a 1} F_{s o c 1}+n_{a 2} F_{s o c 2}+\ldots n_{a i} F_{s o c i}
$$

where the number of individuals using all available patches equals $N$ [eqn (6)].

\section{Solitary provisioners}

A solitary provisioner such as a solitary bee increments its fitness only after it has gathered enough food to provision an offspring (Clark \& Dukas, 1994). We assume that this involves $M$ provisioning trips; if the provisioner survives by the end of that period, it increments its fitness in amount proportional to the total food collected. Hence, the provisioner's expected reproduction per period is

$$
\sigma_{T i}^{M} M \phi_{i}
$$

and its total expected lifetime reproduction is

$$
\mathrm{F}_{\text {soli }}=M \phi_{i}\left(\sigma_{T i}^{M}+\sigma_{T i}^{2 M}+\ldots\right)=\frac{\sigma_{T i}^{M} M \phi_{i}}{1-\sigma_{T i}^{M}} .
$$

At equilibrium, the distribution of solitary provisioners among several patches at varying distances should 
be such that expected lifetime fitness gain at any of the patches for which $n_{i} \geq 1$ should be identical:

$$
\mathrm{F}_{\text {soll }}=F_{\text {sol } 2}=\ldots=F_{\text {soli }}
$$

\section{PROVISIONER DISTRIBUTION IN A}

TWO-DIMENSIONAL FIELD

In many cases, it is pertinent to consider the spatial distribution of provisioners as a function of radial distance from the aggregation. For example, such analysis may be the most practical for evaluating spatial distribution of bees in a large flowering meadow. We present here such two-dimensional analysis for solitary provisioners while assuming that on average, food parameters $(p, e$, and $s)$ are constant with distance and direction from the aggregation. We use here the rate currency, though similar extension to the two-dimensional case can be made with efficiency or lifetime intake.

For the two-dimensional case, we express $s$ and $n$ in units of number of individuals per unit area and denote $x$ as the radial distance from the aggregation. We assume that the forager distribution is radially symmetric about the aggregation, which is located at $x=0$. For simplicity, we also assume no interference, i.e. $q=1$. With these changes, eqns (2) and (3) are slightly modified: now the net rate of loading at a radial distance $x$ from the aggregation is

$$
r(x)=\frac{p e s}{s+n(x)}-c_{p} .
$$

And the net rate of energy delivery is

$$
\mathrm{R}(x)=\frac{L e-\frac{2 x}{v} c_{f}}{\frac{L e}{r(x)}+\frac{2 x}{v}} .
$$

For the solitary provisioners, at equilibrium, $R(x)$ should be identical at all radial distances from the aggregation. Given that all food parameters remain constant with radial distance, $n(x)$ must decline with distance until reaching 0 at distance $D$, defined as the maximal distance the foragers travel. Integrating the distribution $n(x)$ using polar coordinates (where an element of area is of the form $\mathrm{d} A=2 \pi x \mathrm{~d} x)$, results in the total number of provisioners,

$$
\mathrm{N}=2 \pi \int_{0}^{D} n(x) x \mathrm{~d} x
$$

We show in Appendix B that the approximate density profile, $n(x)$, is

$$
\begin{aligned}
n(x)=A-B x, \text { where } A= & \frac{s}{R}(p e-R), \\
& B=\frac{2 p s\left(c_{f}+R\right)}{R v L}
\end{aligned}
$$

and

$$
\mathrm{R} \approx\left(\frac{\pi s p}{12 N}\right)^{1 / 3} e(v L)^{2 / 3}
$$

From this we can also conclude that the total number of foragers in a $1 \mathrm{~m}$ circular band at distance $x$ from the nest is

$$
n_{t o t}(x)=2 \pi x n(x)
$$

where $n(x)$ is given by eqn (16). It also follows from eqn (16) that foragers travel only as far as a distance $D=A / B$.

\section{Results}

PROVISIONER DISTRIBUTION AMONG DISTINCT PATCHES

First, we give the results for the distribution of food provisioners among patches identical in all food parameters except that they are at different distances from the aggregation.

\section{Solitary provisioners}

Here, at equilibrium, the rate of energy intake (or efficiency) of foragers at each patch should be identical. If only two patches are considered, with patch 2 farther than patch 1 , the overall cost of flight to patch 2 is higher. For rates (or efficiencies) to be identical, either the energetic content of food in patch $2\left(e_{2}\right)$, must be higher, or the duration in patch $2\left(t_{p 2}\right)$ must be smaller; for the latter to happen, one of the two patch parameters, production rate $\left(p_{2}\right)$ or size $\left(s_{2}\right)$ must be larger, or the number of foragers at the patch $\left(n_{2}\right)$ should be smaller [eqns (1-3)].

Note that the only difference between the rate and efficiency currencies [eqns (2) and (4)] is the inclusion of parameters for energy expenditure during flight and in the patch $\left(c_{f}\right.$ and $\left.c_{p}\right)$ in the denominator of the efficiency equation. Thus, if $c_{f}=c_{p}$, results using either the rate or efficiency currencies will be similar. The difference in predictions between the two currencies depends on the ratio of rate of energy expenditure in flight and in the patch, $c_{f} / c_{p}$. When $c_{f} \gg c_{p}$, more provisioners will use the closer patch with the efficiency than rate currency. By contrast, if 
$c_{p} \gg c_{f}$, more provisioners will use the closer patch with the rate than efficiency currency.

Figure 1 illustrates numerical solutions [derived from simulations using eqns (2-6)] for the optimal allocation of central place solitary provisioners between two patches identical in all parameters except that patch 2 is farther than patch 1 . Parameter values used are based on empirical values recorded for bees and flowers (Southwick et al., 1981; Wolf et al., 1989; Seeley, 1994). As discussed in the paragraph above, the ratio $c_{f} / c_{p}$ is critical for predictions using the efficiency currency. While energy expenditure in flight can readily be measured, energy expenditure in the patch can vary widely depending on the exact activity of the provisioner; it would be much higher if the provisioner mostly hovers than if it spends its time sitting at a feeder. Although we used here a realistic empirical value of $c_{f}=2 c_{p}$, it is important to recognize the inherent variation for that ratio among and within species. We used three combinations of patch parameters of $p=1, s=25 ; p=1, s=50$; and $p=2, s=25$. With these parameters and when the two patches are at a distance of $500 \mathrm{~m}$ from the colony, average trip duration $\left(t_{f}+t_{p}\right)$ are $75.6 \mathrm{~min}$, $35.5 \mathrm{~min}$, and $16.6 \mathrm{~min}$ respectively. This range of trip duration represents typical lengths reported for bees by various authors (e.g. Ribbands, 1953; Dukas \& Visscher, 1994). Finally, we chose parameter values for patch distance and total number of provisioners so that $R_{i}$ and $r_{i}$ were always positive.

When patch 1 is only $100 \mathrm{~m}$ from the aggregation and patch 2 is up to 10 times farther, the optimal

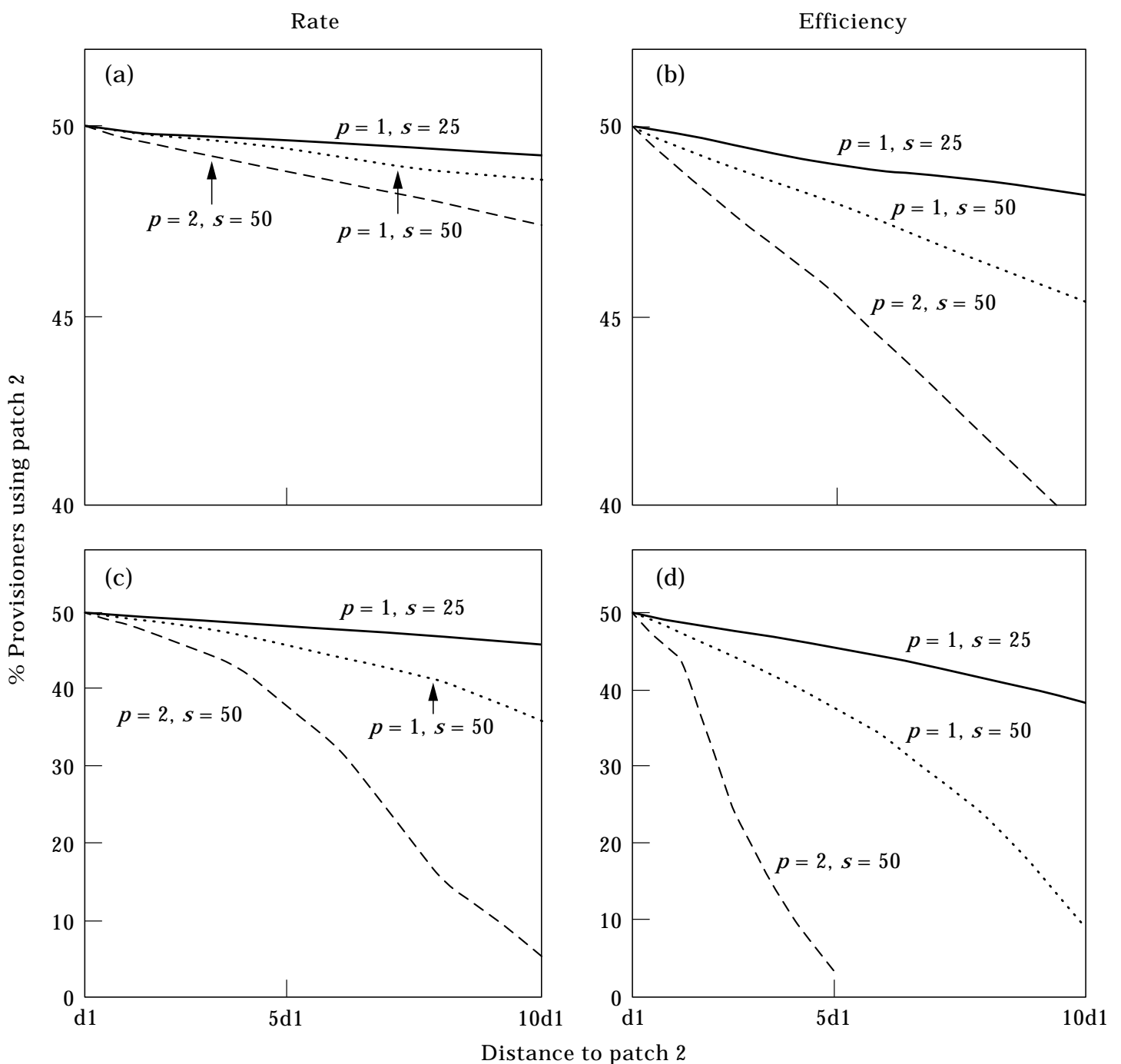

FIG. 1. The equilibrium distribution of solitary provisioners from a single aggregation between two identical food patches located at unequal distances ( $d 1$ and $d 2$ ) from the nest (with 3 sets of $p_{i}, s_{i}$ values). Patch 2 is up to 10 times farther than patch 1 , with patch 1 being $100 \mathrm{~m}(\mathrm{a}, \mathrm{b})$ or $500 \mathrm{~m}(\mathrm{c}, \mathrm{d})$ from the aggregation. Figures (a) and (c) are for the rate currency and (b) and (d) are for efficiency. See Table 1 for other parameter values. 
number of provisioners using patch 2 is only slightly smaller with rate as the currency; but provisioner numbers are up to $20 \%$ smaller with efficiency [Fig. 1(a) and (b)]. When patch 1 is $500 \mathrm{~m}$ from the aggregation and for the rate currency, the proportion of foragers using patch 2 declines sharply with distance only if patch parameter values are relatively large [Fig. 1(c)]. The decline with distance, however, is more dramatic with efficiency (with $c_{f}=2 c_{p}$ ), where no provisioner uses patch 2 beyond $3000 \mathrm{~m}$ for the richest patch values [Fig. 1(d)]. Note that we chose to depict here and throughout the number of provisioners using each patch; this number is usually greater than the number of provisioners observed simultaneously in a patch, because some individuals would be on their way to or from the patch. The relation between these two related numbers appears in eqn (6). The difference between the two quantities must be considered in empirical tests with patches at realistic distances from the aggregation, which may be up to several kilometers for honey bees (e.g. Visscher \& Seeley, 1982).

\section{Load capacity}

Above we assumed that foragers leave a patch only after they have collected a full load. However, it is sometimes optimal for foragers to deliver only partial loads (Schmid-Hempel et al., 1985). To evaluate this issue, we calculated optimal load capacity for individual foragers using an algorithm similar to the one employed by Schmid-Hempel et al. (1985). Briefly, in this algorithm, energy expenditure during flight and in the patch is a function of the provisioner's own weight and its load; hence during loading, energy expenditure increases with each addition to the load. Our results indicate that for patches of very short distances from the nest $(<100 \mathrm{~m})$, optimal loads are well below the maximum load, especially when efficiency is the currency maximized. This agrees with Schmid-Hempel et al. (1985), who used a patch distance of $30 \mathrm{~m}$. For farther distances, optimal load capacity converges to the maximum load used here $(300 \mathrm{j})$. At distances of $100 \mathrm{~m}$ and longer, the optimal load is still lower than the maximum for the smallest rates of food delivery, $\mathrm{r}=0.075 \mathrm{j} / \mathrm{s}$. However, the difference in rate between the optimal and maximum load is smaller than $1 \%$ at $100 \mathrm{~m}$, and differences in efficiency between the optimal and maximum load are only $7 \%$ at $100 \mathrm{~m}$, and $1.7 \%$ at $500 \mathrm{~m}$. Given that the differences between optimal and maximal load are so small even for the shortest distances considered here, our assumption of maximum load is well justified, at least for the realistic bee parameters used here.

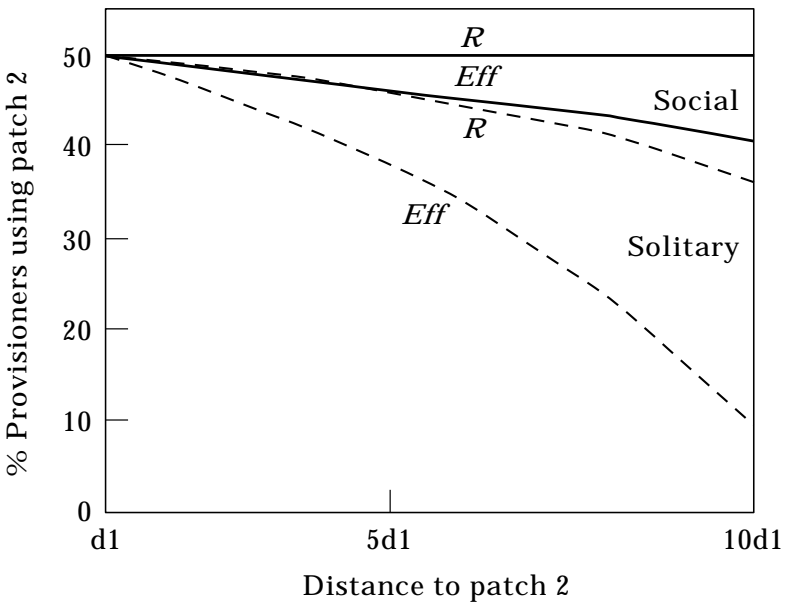

FIG. 2. Comparison of the equilibrium distribution of solitary (broken line) and social (continuous line) provisioners from a single aggregation between two food patches. The patches are identical (with $p_{i}=1, s_{i}=50$ ) except that patch 2 is up to 10 times farther than patch 1 , which is $500 \mathrm{~m}$ away from the aggregation. Results are for rate $(R)$ and efficiency $(E f f)$. Other parameter values are as in Fig. 1.

\section{Social versus solitary provisioners}

The outcome of the optimal provisioning strategy for a solitary provisioner would be that at equilibrium, all provisioners have identical net rate of gain. Such a condition does not hold for the social provisioners, where the colony's net rate of gain is maximized. It can be shown analytically that this difference in optimization schemes produces distinct spatial distribution of provisioners, with more social provisioners exploiting the farther patch (Appendix A). Numerical examples based on eqns (5) and (7) show that the difference in strategies is less than $10 \%$ when rate is maximized, and up to $30 \%$ for efficiency (Fig. 2).

\section{BALANCING FORAGING GAIN AND PREDATION RISK}

As with efficiency [eqn (4)], outcomes while maximizing lifetime fitness depend on the ratio between two parameters; this time, the parameter ratio is instantaneous mortality rate in flight over instantaneous mortality rate in the patch, $\mu_{f} / \mu_{p}$. Numerical examples based on eqns (8-12) show that when $\mu_{f}=\mu_{p}$, provisioner distribution while using lifetime gain is similar to that obtained with rate (Fig. 3). That is, there is relatively small reduction in number of provisioners using the more distant patch even when it is 10 times farther (with $p_{i}=1$ and $s_{i}=50$ ). When $\mu_{f}=2 \mu_{p}$, results with lifetime gain are similar to the ones using efficiency with a parallel ratio $c_{f}=2 c_{p}$. That is, the reduction in number of provisioners using the farther patch is larger than with rate. However, unlike efficiency, where there is 
some empirical foundation for using a ratio of $c_{f}=2 c_{p}$, the ratio of mortality rates is yet unknown. Hence, we considered also a third possibility, with $\mu_{f}=0.5 \mu_{p}$. Here the proportion of provisioners using the farther patch is slightly reduced for solitary provisioners and slightly increased for social provisioners (Fig. 3).

\section{Social versus solitary provisioners}

As before, the difference in optimization schemes produces distinct spatial distributions of provisioners, with more social provisioners exploiting the farther patch. The differences in strategies is rather small with $\mu_{f}=\mu_{p}$ and $\mu_{f}=0.5 \mu_{p}$, but it is as high as $30 \%$ with $\mu_{f}=2 \mu_{p}$ (Fig. 3).

\section{PROVISIONER DISTRIBUTION IN A TWO DIMENSIONAL FIELD}

The density of solitary provisioners declines linearly with distance when average food parameters do not change with distance and direction from the aggregation and rate is maximized. The overall number of provisioners initially increases, peaks at half the maximal distance, $D$, and then declines to 0 at $D$ (Fig. 4).

\section{Discussion}

\section{ASSUMPTIONS AND CURRENCIES}

The spatial distribution of provisioners that share a central nesting site presents some balance between the cost of foraging closer, where competition with other provisioners is higher, and the cost of traveling farther. Such costs may be expressed as overall trip

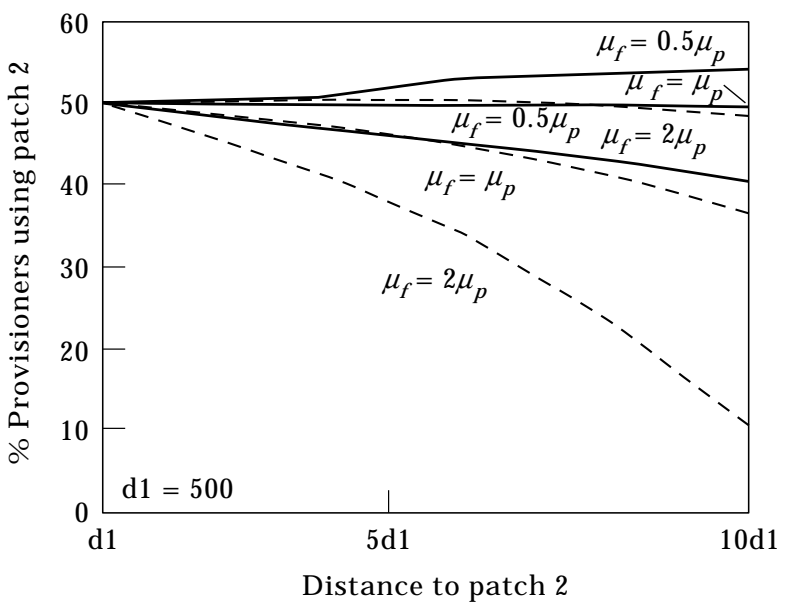

FIG. 3. Comparison of the equilibrium distribution of solitary $(--)$ and social (-) provisioners when lifetime gain is maximized, with three ratios of instantaneous mortality rate in flight over instantaneous mortality rate in the patch, $\mu_{f} / \mu_{p}$. The numerical solutions are based on eqns (8-12), with parameter values as in Fig. 2 and Table 1.

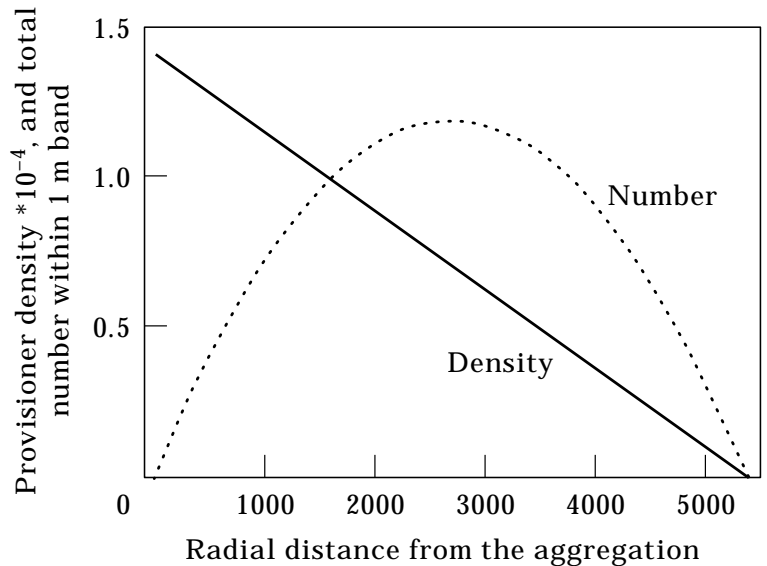

FIG. 4. The equilibrium distribution of solitary provisioners from a single aggregation in a two dimensional field of food as a function of radial distance from the aggregation. The continuous line depicts provisioner density, $n(x)$, and the broken line shows total number of provisioners within a band of 1 meter, $n_{t o t}(x)$. Parameter values are $N=5000, p=2, s=10^{-5}$, and other parameter values as in Table 1. With these parameters, the equilibrium rate of energy intake is $0.13 \mathrm{j} \mathrm{s}^{-1}$.

duration, rates of energy expenditure during travel and during foraging, or mortality rates during travel and during foraging. Although that general notion has been recognized (Hamilton \& Watt, 1970; Covich, 1976; Taylor, 1977; Anderson, 1978; Bartholdi et al., 1993), no previous study has considered the overall factors involved. Rather, recent foraging studies have focused either on the behavior of a single central place forager or on models based on ideal free distribution.

A crucial component of our model is the assumptions about food production and exploitation [eqn (3)]. We chose to present a renewable resource, which is realistic for many systems and especially for flowers and bees on which we based our numerical examples. That resource has a per unit energy production rate and an overall size or density. This can be measured empirically as the number of provisioners at which gross consumption rate is half its maximum rate $\left(p_{i} e_{i} / 2\right)$ if interference is negligible. Interference among individuals is likely to occur beyond a certain density (Stillman et al., 1996) and it can be quantified using eqn (3).

Another key feature is the choice of currency to be maximized. Although rate still dominates foraging theory (Stephens \& Krebs, 1986; Krebs \& Davies, 1991), it is now clear that mortality probabilities associated with various activities strongly affect foraging choice (e.g. Milinski \& Heller, 1978; Lima \& Dill, 1990; Sih, 1992). To include mortality in our model, we had to choose values for the ratio of mortality rate during flight and in the patch. 
TABLE 1

\begin{tabular}{|c|c|c|}
\hline$\overline{\mathrm{L}}$ & Load capacity & $40 \mu \mathrm{l}$ (Seeley, 1994) \\
\hline$e_{i}$ & Energy content per unit load volume & $7.5 \mathrm{j} \mu \mathrm{l}^{-1}$ (Southwick et al. 1981) \\
\hline$t_{p i}$ & Total time spent in patch $i$ & \\
\hline$t_{f i}$ & Total time spent flying to patch $i$ & \\
\hline & Distance to patch $i$ & \\
\hline$v$ & Flight velocity & $7 \mathrm{~m} \mathrm{~s}^{-1}$ (Seeley, 1994) \\
\hline$c_{f}$ & Cost of flight per time unit & $0.05 \mathrm{j} \mathrm{s}^{-1}$ (Wolf et al. 1989a, b) \\
\hline$r_{i}$ & Net rate of loading in patch $i$ & \\
\hline$p_{i}$ & Gross maximum per capita rate of loading in patch & 1 or $2 \mathrm{j} \mathrm{s}^{-1}$ (Southwick et al, 1981) \\
\hline & Parameter related to patch size & 25 or 50 \\
\hline$n_{i}$ & Number of provisioners foraging simultaneously in patch $i$ & \\
\hline & Interference competition & 1 \\
\hline$c_{p}$ & Energetic cost of loading in the patch & $0.025 \mathrm{j} \mathrm{s}^{-1}$ (Wolf et al. 1989a, b) \\
\hline$n_{a i}$ & Number of provisioners using patch $i$ & \\
\hline$N$ & Total number of active foragers in the aggregation & 500 or 5000 \\
\hline$\mu_{f}$ & Probability of mortality per time period during flight & $0.5,1$, or $2 \mu_{p}$ \\
\hline & Probability of mortality per time period while in the patch & $2.510^{-6} \mathrm{~s}^{-1}$ \\
\hline$M$ & Number of trips required by a solitary provisioner to gather food for an offspring & 15 \\
\hline
\end{tabular}

*Some parameter values are rough or rounded estimates.

Although there has been significant interest in the association between foraging effort and mortality rate (Wolf \& Schmid Hempel, 1989a, b), no data exist for the ratio of mortality rates during the two distinct activities of traveling to and foraging in a patch. Such information can readily be gathered at least for honey bees by monitoring departures and arrivals of marked bees at the hive and at distant feeders or flower patches.

It is intriguing that when the ratio of mortality rates is identical to the ratio of energy expenditures in travel and in the patch, outcomes using the lifetime fitness algorithm [eqns (8-12)] are identical to results using efficiency [eqn (4); Fig. 2 vs. Fig. 3]. Since Schmid Hempel et al. (1985) found that foraging decisions of honey bees were better described by efficiency than rate, other empirical studies have confirmed this outcome (e.g. McLaughlin \& Montgomerie, 1990; Welham \& Ydenberg, 1993; Seeley, 1994). Efficiency, however, is an empirically based algorithm that has not been derived from first principles. Houston et al. (1988) argued that when mortality increases with effort, the currency maximized should be between rate and efficiency. Our simpler algorithm for lifetime energy intake shows more clearly why, under certain conditions, foraging decisions based on balancing energy intake and mortality risk may appear as decisions based on maximizing efficiency.

Note, however, that we assumed that a provisioner maximizes its life time food intake. This is legitimate for solitary provisioners with non-overlapping generations, and for social workers of perennial colonies at times they do not undergo rapid growth; for example, honey bees may devote a large portion of the summer to storing food for the winter. Neither our algorithm nor the one used by Houston et al. (1988) is appropriate for annual or perennial colonies experiencing exponential growth. In that case, a fitness measure similar to the intrinsic rate of growth in populations with overlapping generations $(r)$ is required.

Ydenberg et al. (1994) suggested that limitation on the rate of energy assimilation can explain efficiency maximization. This legitimate explanation requires quantification of actual energy assimilation by foragers to verify that it is indeed a constraint driving foraging behavior. In addition, predictions using efficiency maximization should be compared with predictions using lifetime energy intake if one is to establish either currency as the one employed by animals. That is, first, assumptions used for either currency must be quantified; and second, given that both currencies maximize gain over cost, their predictions should be compared to each other and not only to predictions with rate, which are sometimes quite different. Of course, the use of any of the three currencies considered here may be appropriate depending on the species studied, ecological circumstances, and an animal's physiological state.

\section{SOCIAL VS. SOLITARY PROVISIONERS}

Our analyses suggest that the spatial distribution of provisioners belonging to a social aggregation should differ from that of solitary provisioners. Technically, maximization of energy intake (or any other currency) should be at the colony level for social provisioners and at the individual level for solitary provisioners. The outcome of individual maximization is that at equilibrium, all individuals have 
identical intake. This condition need not hold for a social colony, where some individuals, by collecting food farther, reduce competition with kin closer to the aggregation. Even though per capita gain of such individuals is lower, the overall colony gain is higher (Figs 2 and 3; Appendix A). This difference between solitary and social provisioners provides another fitness advantage of sociality (Wilson, 1971; Michener, 1974; Clark \& Dukas, 1994). Of course, it remains to be empirically evaluated whether social provisioners are indeed sensitive to such variation in maximization schemes. At the proximate level, this can readily be achieved by an increased tendency of social provisioners to collect food farther from the colony than solitary provisioners.

\section{PROVISIONER DISTRIBUTION IN A TWO DIMENSIONAL FIELD}

Our results for distinct patches can readily be extended to two dimensions as illustrated here for the rate currency (Fig. 4). This two-dimensional analysis may be more relevant in many natural or semi-natural conditions. For example, predictions of our model can be tested in a large cultivated field of flowers with a single aggregation of solitary or social bees in its center. Applied studies on honey bees have provided some data on the two-dimensional distribution of foragers in agricultural fields (e.g. Lee, 1961; Levin, 1961; Gary et al., 1978). But this information is not suitable for evaluating our model, because the above studies involved numerous colonies, some of which were monitored as a part of the experiment while others were not. Nevertheless, such empirical data illustrate the feasibility of evaluating assumptions and predictions of our model under realistic yet controlled two-dimensional field settings.

\section{THE MAXIMUM DISTANCE TRAVELED TO COLLECT FOOD}

It is of interest to evaluate how far foragers should go from their central place to collect food. The simplest answer can be derived from the numerator of eqn (2), by solving for the maximum distance for which net energy gain is positive. For the bee parameter values used here $\left(L e_{i}=300, v=7\right.$, and $\left.c_{f}=0.05\right), \mathrm{d}_{\max }=21 \mathrm{~km}$. The parallel answer for the two-dimensional case is $\mathrm{d}_{\max }=D=A / B$ where $A$ and $B$ are defined in eqn (16) (see also Appendix B). For the parameter values used for Fig. $4, D=5,375 \mathrm{~m}$. A more realistic answer for maximal travel distance would consider the relative rates of energy gain as one goes farther from the central place; these rates depend on food quality and abundance, on the number of foragers in the aggregation, and on the distribution of foragers from neighboring aggregations. Note that even if the rate of energy gain within several $\mathrm{km}$ from an aggregation is relatively low, provisioners may decide not to fly farther, but instead, wait in the nest until forage conditions improve. It appears that honey bees do indeed reduce the number of foragers under low forage availability and increase the work force when forage is rich (Seeley, 1996). To understand such level of decision making either in solitary or social foragers, one must consider lifetime fitness and the way it is affected by alternative strategies at low food availability, such as waiting in the nest for a period of several days, foraging at farther distances for rich food, or foraging at closer distances for poor food. Another issue that needs consideration is the discovery of food patches if these are difficult to find at a distance. Unfortunately, we do not yet know the typical detection distance even for sources such as floral patches. It is likely, though, that detection distance greatly varies among plant species, patch sizes and weather conditions (see Seeley, 1987, 1996).

\section{CAVEATS}

Our analyses provide an important link between individual behavior as expressed in models of central place foraging and its relation to population level distribution as analysed in ideal free distribution models. Still, we focused here only on a restricted set of basic issues and there are a few additional factors requiring close examination.

\section{Inter-aggregation interactions}

So far, we have only considered a single aggregation of provisioners because we think it is crucial to establish an empirically sound working model before proceeding to multi-colony interactions. Our assumptions and predictions for rate of energy intake under exploitation and interference competition, currencies maximized, and maximization schemes for solitary and social provisioners can all be tested with a single aggregation in either a distinct patch or in a two-dimensional settings. Once newly gathered data allow us to narrow down alternatives considered here, it will be easier to evaluate the spatial distribution of provisioners from more than one aggregation.

\section{Information and stochasticity}

We implicitly assumed that provisioners have perfect knowledge of location and quality of food sources, provisioner density, and mortality risks. In reality, such information must be gathered and updated constantly because of spatial and temporal changes (Stephens \& Krebs, 1986; Bernstein et al., 1991). To include such issues of information, we need 
to know more about a forager's rate of acquisition of information.

In sum, our spatially explicit models provide testable new predictions for the spatial distribution of numerous solitary or social food provisioners that share a central place. Hence these models are relevant for a variety of well studied species of birds and bees. By including effects of distance from the central place and the fundamental factors of exploitation and interference competition, we provide a link between the somewhat separate models of central place foraging by a single individual and "ideal free distribution" of many foragers.

We thank an anonymous referee, R. Ydenberg, and other members of the Behavioural Ecology Group at Simon Fraser University for comments, and NSERC Canada operating grants to LK and C. Clark for financial support.

\section{REFERENCES}

ANDERSSON, M. (1978). Optimal foraging area: size and allocation of search effort. Theor. Pop. Biol 13, 397-409.

Bartholdi, J. J., Seeley, T. D., Tovey, G. A. \& Vande Vate, J. H. (1993). The pattern and effectiveness of forager allocation among flower patches by honey bee colonies. $J$. theor. Biol. 160, 23-40. Batra, S. W. T. (1984). Solitary bees. Sci. Am. 250(2), 86-93.

Bernstein, C., Kacelnik, A. \& Krebs, J. R. (1991). Individual decisions and the distribution of predators in a patchy environment. II. The influence of travel costs and structure of the environment. J. Anim. Ecol. 60, 205-225.

Brown, C. R. \& Brown, M. B. (1996). Coloniality in the Cliff Swallow. Chicago: University of Chicago Press.

Camazine, S. \& Sneyd, J. (1991). A model of collective nectar source selection by honey bees: self-organization through simple rules. J. theor. Biol. 149, 547-571.

Clark, C. W. \& Dukas, R. (1994). Balancing foraging and antipredator demands: an advantage of sociality. Am. Natur. 144, 542-548.

Covich, A. P. (1976). Analyzing shapes of foraging areas: some ecological and economic theories. Ann. Rev. Ecol. System. 7, 235-257.

DUKAS, R. \& VisSCHER, P. K. (1994). Lifetime learning by foraging honey bees. Anim. Behav. 48, 1007-1012.

FreTwELL, S. D. \& LuCAS, J. H. J. (1970). On territorial behaviour and other factors influencing habitat distribution in birds. Acta Biotheor. 19, 16-36.

Gary, N. E., Witherell, P. C. \& Lorenzen, K. (1978). The distribution and foraging activities of common Italian and "Hy-Queen" honey bee during alfalfa pollination. Env. Ent. 7, 233-240.

Gaston, A. J., Chapdelaine, G. \& Noble, D. G. (1983). The growth of Thick-billed Murre at colonies in Hudson Strait: inter-and intra-colony variation. Can. J. Zool. 61, 2465-2475.

Gilliam, J. F. \& Fraser, D. F. (1987). Habitat selection under predation hazard: test of a model with foraging minnows. Ecology 68, 1856-1862.

Hamilton, W. J. \& Watt, K. E. F. (1970). Refuging. Ann. Rev. Ecol. System. 1, 263-286.

Hamilton, W. J., Gilbert, W. M., Heppner, F. H. \& Planck, R. J. (1967). Starling roost dispersal and a hypothetical mechanisms regulating rhythmical animal movement to and from dispersal centers. Ecology 48, 825-833.

Hassell, M. P. (1978). The Dynamics of Arthropod Predator-Prey systems. Princeton, NJ: Princeton University Press.
Holldobler, B. \& Wilson, E. O. (1990). The Ants. Cambridge, MA.: Harvard University Press.

Houston, A. I., Schmid-Hempel, P. \& Kacelnik, A. (1988). Foraging strategy, worker mortality, and the growth of the colony in social insects. Am. Natur. 131, 107-114.

Kacelnik, A., Krebs, J. R. \& Bernstein, C. (1992). The ideal free distribution and predator-prey populations. Trends Ecol. Evol. 7 , $50-55$.

Krebs, J. R. \& Davies, N. B. (eds) (1991). Behavioural Ecology. London: Blackwell.

LEE, W. R. (1961). The nonrandom distribution of foraging honey bees between apiaries. J. Econ. Ent. 54, 928-933.

LEvin, M. D. (1961). Distribution of foragers from honey bee colonies placed in the middle of a large field of alfalfa. $J$. Econ. Ent. 54, 431-434.

Lima, S. L. \& Dill, L. M. (1990). Behavioral decisions made under the risk of predation: a review and prospectus. Can. J. Zool. 68 , 619-640.

Mclaughlin, R. L. \& Montgomerie, R. D. (1990). Flight speeds of parental birds feeding dependent nestlings: maximizing foraging efficiency or food delivery rate? Can. J. Zool. 68, 2269-2274.

Michener, C. D. (1974). The Social Behavior of the Bees. Cambridge, MA: Harvard University Press.

Milinski, M. \& PARKer, G. A. (1991). Competition for resources. In: Behavioral Ecology. (J. R. Krebs \& N. B. Davies, eds), pp. 137-168. London: Blackwell.

Orians, G. H. \& Pearson, N. E. (1979). On the thoery of central place foraging. In: Analysis of Ecological Systems. (D. J. Horn, R. D. Mitchell \& G. R. Stairs eds), pp. 154-177. Columbus: Ohio State University Press.

Pleasants, J. M. (1981). Bumblebee response to variation in nectar availability. Ecology 62, 1648-1661.

Possingham, H. P. (1992). Habitat selection by two species of nectarivore: habitat quality isolines. Ecology 73, 1903-1912.

RibBANDS, C. R. (1953). The Behavior and Social Life of Honeybees. London: Bee Research Association.

Schmid-Hempel, P., Kacelnik, A. \& Houston, A. I. (1985). Honeybees maximize efficiency by not filling their crop. Behav. Ecol. Soc. 17, 61-66.

SCHOENER, T. W. (1979). Generality of the size-distance relation in models of optimal feeding. Am. Natur. 114, 902-914.

SeELey, T. D. (1987). The effectiveness of information collection about food sources by honey bee colonies. Anim. Behav 35, $1572-1575$.

SEELEY, T. D. (1994). Honey bee foragers as sensory units of their colonies. Behav. Ecol. Soc. 34, 51-62.

Seeley, T. D. (1996). The Wisdom of the Hive. Cambridge, MA: Harvard University Press.

Seeley, T. D., Camazine, S. \& Sneyd, J. (1991). Collective decision making in honey bees: how colonies choose among nectar sources. Behav. Ecol. Soc. 28, 277-290.

SiH, A. (1980). Optimal behavior: can foragers balance two conflicting demands? Science 210, 1041-1043.

Sit, A. (1992). Prey uncertainty and the balancing of antipredator and feeding needs. Am. Natur. 139, 1052-1069.

Southwick, E. E., Loper, G. M. \& SADwick, S. E. (1981). Nectar production, composition, energetics and pollinator attractiveness in spring flowers of western New York. Am. J. Botany 68, 994-1002.

Stephens, D. W. \& Krebs, J. (1986). Foraging Theory. Princeton NJ: Princeton University Press.

Sutherland, W. J. (1996). From Individual Behaviour to Population Ecology. New York: Oxford University Press.

TAYLOR, F. (1977). Foraging behavior of ants: experiments with two species of Myrmecine ants. Behav. Ecol. Soc. 2, 147-167.

TAYLOR, F. (1978). Foraging behavior of ants: theoretical considerations. J. theor. Biol. 71, 541-565.

Visscher, P. K. \& SeEley, T. D. (1982). Foraging strategy of honeybee colonies in a temperate deciduous forest. Ecology 63, 1790-1801. 
Welham, C. V. J. \& Ydenberg, R. C. (1993). Efficiency-maximizing flight speeds in parent black terns. Ecology 74, 1893-1901.

Werner, E. E. \& Gilliam, J. F. (1984). The ontogenetic niche and species interaction in size structured populations. Ann. Rev. Ecol. System. 15, 393-425.

Wilson, E. O. (1971). The Insect Societies. Cambridge, MA: Harvard University Press.

WOLF, T. J. \& SCHMID-HemPel, P. (1989a). Extra loads and foraging life span in honeybee workers. J. Anim. Ecol. 58, 943-954.

Wolf, T. J., Schmid-Hempel, P., Ellington, C. P. \& Stevenson, R. D. (1989b). Physiological correlates of foraging efforts in honey bees: oxygen consumption and nectar load. Functional Ecol. 3, 417-424.

YDENBERG, R. C. (1997). Behavioral decisions about foraging and predator avoidance. In: Cognitive Ecology: The Evolutionary Ecology of Information Processing and Decision Making. (Dukas, R., ed.). Chicago: University of Chicago Press (in press).

YdenberG, R. C. \& Schmid-Hempel, P. (1994). Modelling social insect foraging. Trends in Ecology and Evolution 9, 491-493.

Ydenberg, R. C., Welham, C. V. J., Schmid-Hempel, R., Schmid-Hempel, P. \& Beauchamp, G. (1994). Time and energy constraints and the relationships between currencies in foraging theory. Behav. Ecol. 5, 28-34.

\section{APPENDIX A}

In order to analytically compare the Social and Solitary forager case, we used a simplified version of the expression for rate. We defined the basic rate of energy uptake associated with patch $i, R_{i}$ by the formula

$$
R_{i}=\frac{a_{i}}{g\left(n_{i}\right)}
$$

where

$$
a_{i}=L e_{i}-2 C_{f i}=L e_{i}-2 c_{f} \frac{d_{i}}{v},
$$

is the uptake of energy discounted by the energy spent in travelling to the patch, and

$$
g\left(n_{i}\right)=t_{p i}+t_{f i} \approx t_{p i}=\frac{L}{C\left(n_{i}\right)},
$$

is the time spent in the patch given that the rate of energy gain is $C\left(n_{i}\right)$. [See eqn (1) in main text]. We assumed, further, that

$$
C\left(n_{i}\right)=\frac{p_{i} e_{i} s_{i}}{s_{i}+n_{i}^{q}},
$$

where $n_{i}$ is the number of individuals in the given patch. (The simplification in the above expression consists in neglecting the time spent in travel and the energetic cost of loading.)

For convenience of notation, we here set $e_{i}=1$. We also assume that $q=1$. We are considering the case of two patches whose sizes and qualities are identical $\left(s_{1}=s_{2}=s, p_{1}=p_{2}=p\right)$, but with Patch 1 closer to the nest. Thus, $d_{1}<d_{2}$ and $a_{1}>a_{2}$. Further, $a_{1}, a_{2}>0$ can be assumed. A comparison of the Solitary and
Social cases for the two patch problem can be made as follows.

\section{Solitary Case}

We must find a way of subdividing the total population, $N$ into numbers of individuals in the two patches, i.e., $n_{1}+n_{2}=N$ such that $R_{1}=R_{2}$. This implies

$$
\frac{a_{1}}{g\left(n_{1}\right)}=\frac{a_{2}}{g\left(n_{2}\right)} .
$$

and leads to the equation

$$
\frac{a_{1}}{\left(s+n_{1}\right)}=\frac{a_{2}}{\left(s+N-n_{1}\right)} \text {. }
$$

Solving this equation for $n_{1}$ results in:

$$
n_{1}=N \frac{\left(a_{1}-a_{2}\right) \frac{s}{N}+a_{1}}{a_{1}+a_{2}}
$$

It is useful to express this solution to eqn (A.6) in terms of the fraction, $f$, of the population in patch 1

$$
f=\frac{n_{1}}{N}, 1-f=\frac{n_{2}}{N} .
$$

We further define the ratio of net energy gains,

$$
y=\frac{a_{1}}{a_{2}} .
$$

By simplifying eqn (A.6) and re-expressing it in terms of the above ratios, we find that the fraction of foragers in Patch 1 in the solitary case, $f$, can be expressed in the form:

$$
f=\frac{y+(y-1) \frac{s}{N}}{(y+1)} .
$$

Note that $f$ has been expressed in terms of the ratio of energy gains, $y$.

\section{Social Case}

In the social case, we must find values of $n_{1}, n_{2}$ (with $n_{1}+n_{2}=N$ ), that maximize the Total rate of energy uptake by the whole population

$$
R=\frac{n_{1} a_{1}+n_{2} a_{2}}{n_{1} g\left(n_{1}\right)+n_{2} g\left(n_{2}\right)} .
$$

Using the expressions for $g$ for the two (identical) patches, and the notation $f$ defined above, eqn (A.11) can be expressed in the form

$$
R=\left(\frac{p s}{N L}\right) \frac{\left(a_{1}-a_{2}\right) f+a_{2}}{-2 f(1-f)+\left(\frac{s}{N}+1\right)} .
$$


From this equation, we can make these observations:

(1) the Total Rate can be expressed in terms of ratios of the individuals.

(2) The value of the parameter $p$ appears only in the braces multiplying the expression shown in eqn (A.12) above. Thus, $p$ can affect the scaling of the total rate, but not the optimal value of the fraction in patch $1, f$. Thus, if the patches are identical, their quality does not alter the distribution of individuals. Note that the value of $L$ affects the quantities $a_{1}, a_{2}$ which appear in the fractional expression and so this statement is not true for $L$.

(3) We have used Patch 1 as the reference patch, but clearly, the same expression could have been rewritten in terms of the proportion going to Patch 2.

To find the optimal individual allocation, we must find the maximum value of $R$ with respect to the unknown $f$. Setting

$$
\frac{\mathrm{d} R}{\mathrm{~d} f}=0
$$

leads, after some simplification, to a quadratic equation for $f$,

$$
a f^{2}+b f-c=0
$$

where

$$
\begin{aligned}
a=2\left(a_{1}-a_{2}\right), b & =4 a_{2}, \\
c & =\frac{s}{N}\left(a_{1}-a_{2}\right)+\left(a_{1}+a_{2}\right) .
\end{aligned}
$$

(All of the above coefficients are positive according to our comments about the relative magnitudes of $a_{1}$ and $a_{2}$.) The solution to this quadratic equation is

$$
f=\frac{-a_{2}+\frac{\sqrt{2}}{2} \sqrt{\left(a_{2}^{2}+a_{1}^{2}\right)+\frac{s}{N}\left(a_{1}-a_{2}\right)^{2}}}{\left(a_{1}-a_{2}\right)} .
$$

The solution is expressed in a more compact form in terms of the ratio of the energy gains for the two patches, $y=a_{1} / a_{2}$, by dividing numerator and denominator of eqn (A.16) by $a_{2}$. The result is

$$
f=\frac{-1+\frac{\sqrt{2}}{2} \sqrt{\left(1+y^{2}+\frac{s}{N}(1-y)^{2}\right.}}{(y-1)} .
$$

\section{Comparison of the Two Systems}

By previous remarks about the relative magnitudes of $a_{1}, a_{2}$, we know that $y>1$, and $(y-1)$ is positive.
We can compare eqns (A.10) and (A.17) directly, in a limiting case that $y$ is large, i.e. that Patch 1 is much closer than Patch 2. We find that for $y \gg 1$,

$$
f_{\text {social }} \approx \frac{1}{\sqrt{2}} \sqrt{1+\frac{S}{N}}
$$

whereas

$$
f_{\text {solitary }} \approx 1+\frac{s}{N} .
$$

Since the quantity inside the radical is larger than 1, clearly, for large $y, f_{\text {solitary }}>f_{\text {social }}$, i.e. the proportion of individuals that go to the closer patch (Patch 1) is higher in the solitary case than in the social case.

\section{APPENDIX B}

The density profile for solitary foragers in a two-dimensional field can be derived in the following way: We consider the two equations

$$
\begin{gathered}
R=\frac{\mathscr{L}-\gamma_{1} x}{\frac{\mathscr{L}}{r}+\gamma_{2} x} \\
r=\frac{K}{s+n(x)}-\varepsilon
\end{gathered}
$$

where the parameters are

$$
\mathscr{L}=L e, \gamma_{1}=\frac{2 c_{f}}{v}, \gamma_{2}=\frac{2}{v}, K=\text { pes }, \varepsilon=c_{p}
$$

We consider the approximation $\varepsilon=c_{p} \approx 0$ which greatly simplifies the problem. (The full case can also be treated, but the results are more cumbersome, and will not be described here.) In the solitary forager case, the rate $R$ is the same at every distance from the aggregation but its value is not a priori known. We can find a relationship between $1 / r$ and $x$ by rearranging the first equation. The result is

$$
\frac{1}{r}=\frac{1}{R}\left(1-\frac{\left(\gamma_{1}+\gamma_{2} R\right) x}{\mathscr{L}}\right)
$$

We also note from the second equation (with $\varepsilon \approx 0$ ) that

$$
\frac{1}{r} \approx \frac{(s+n)}{K}
$$

Equating the two expressions for $1 / r$ and solving for $n(x)$ leads to the result

$$
n(x)=\frac{K}{R}\left(1-\frac{s R}{K}-\frac{\left(\gamma_{1}+\gamma_{2} R\right) x}{\mathscr{L}}\right)
$$


which, in terms of the original parameters is

$$
n(x)=\frac{p e s}{R}\left(1-\frac{R}{p e}-\frac{2\left(c_{f}+R\right) x}{v L e}\right) .
$$

For this density profile to make sense, we must have a positive density close the the nest (when $x=0$ we require $n>0$ ). This imposes the restriction

$$
p e>R \text {. }
$$

We now observe that the density profile is a function whose form is

$$
n(x)=A-B x
$$

where, after some simplification, the quantities $A, B$ are

$$
A=\frac{s}{R}(p e-R), B=\frac{2 p s\left(c_{f}+R\right)}{R v L} .
$$

Thus, in the approximate limit that $c_{p}=0$, the density falls off linearly with distance from the nest. This density profile is meaningful only up to the distance $x=D$ at which $n=0$. This distance, which corresponds to the farthest distance of travel and might be called a "domain size" is given by

$$
D=\frac{A}{B}=\frac{v L(p e-R)}{2 p\left(c_{f}+R\right) .}
$$

The above results contain the parameter $R$ which represents the rate of energy gain actually attained at each location. Though $R$ is the same at every distance $x$, its value is not known ahead of time. The value of
$R$ can be found if we specify the total size of the population, $N$. We have

$$
\begin{aligned}
N=2 \pi \int_{0}^{D} n(x) x \mathrm{~d} x=2 \pi \int_{0}^{D}\left(A x-B x^{2}\right) \mathrm{d} x \\
=2 \pi\left(A \frac{D^{2}}{2}-B \frac{D^{3}}{3}\right)
\end{aligned}
$$

If we substitute the value of $D$ into the result, we find that

$$
N=\frac{\pi}{3} \frac{A^{3}}{B^{2}}=\frac{\pi}{3}\left(\frac{s}{R}(p e-R)\right)^{3}\left(\frac{R v L}{2 p s\left(c_{f}+R\right)}\right)^{2} .
$$

The expression linking $N$ to $R$ is cumbersome, and it would be difficult to solve for $R$ in terms of $N$ directly, unless we simplify the problem further. It we make the further assumption that $R>c_{f}$ and that $p e>R$ then we can approximate $N$ by

$$
N \approx \frac{1}{R^{3}} \frac{\pi s p e^{3}}{3}\left(\frac{v L}{2}\right)^{2}
$$

which allows us to conclude that

$$
R \approx\left(\frac{\pi s p}{12 N}\right)^{1 / 3} e(v L)^{2 / 3}
$$

We now have the previously unknown value of $R$ expressed in terms of the total population size and other parameters of the problem. We can use this expression in the coefficients $A$ and $B$ so that the function $n(x)$ is fully determined. 